\title{
Lindeberg-Feller theorems on Lie groups
}

\author{
G. Pap* \\ Institute of Mathematics, Lajos Kossuth University \\ H-4010 Debrecen, Pf.12, Hungary
}

\section{Introduction}

The author has proved in [4] several versions of Lindeberg-Feller type central limit theorems on the Heisenberg group, that is, necessary and sufficient conditions for a triangular array $\left(\mu_{n \ell}\right)_{\ell=1, \ldots, k_{n} ; n \geqslant 1}$ of probability measures on the Heisenberg group to converge to a Gauss measure. Lindeberg type condition was used in the form

$$
\lim _{n \rightarrow \infty} \sum_{\ell=1}^{k_{n}} \int_{|x| \geqslant \varepsilon}|x|^{2} \mu_{n \ell}(d x)=0 \quad \text { for all } \varepsilon>0,
$$

where $x \mapsto|x|$ denotes a fixed homogeneous norm on the Heisenberg group.

Professor E. Siebert suggested a way to prove similar results for a Lie group $G$. The main step is the generalization of Lemma 1 in Pap [4] for an arbitrary Lie group $G$, which gives an estimation for the Fourier transform of a probability measure on $G$ in terms of integrals of local coordinates and a Hunt function, see Section 3.

This lemma makes it possible to apply the accompanying Poisson system of a triangular system $\left(\mu_{n \ell}\right)_{\ell=1, \ldots, k_{n} ; n \geqslant 1}$ of probability measures on $G$ in the usual way, see Section 4 .

Section 5 is devoted to Lindeberg-Feller type theorems on an arbitrary Lie group. Section 6 contains the important special case of stratified Lie groups, where the usual form of the Lindeberg-Feller theorem can be obtained.

\section{Notations}

If $H$ is a subset of a nonvoid set $I$ then $C H$ denotes the complement of $H$ in $I$ and $1_{H}$ the indicator function of $H$.

\footnotetext{
${ }^{*}$ Research work supported by the Alexander von Humboldt Foundation and completed at the University of Tübingen, Germany

AMS 1980 subject classifications: Primary 60B15; secondary 60F05
} 
Let $G$ be a Lie group of dimension $m$. Let $\mathfrak{U}(e)$ be the system of all measurable neighbourhoods of the identity $e$ in $G$. By $\mathbf{e}^{b}(G)$ we denote the space of real valued bounded continuous functions on $G$ furnished with the supremum norm $\|\cdot\|_{\infty} \cdot \mathfrak{C}^{0}(G)$ denotes the subspace of functions vanishing at infinity. By $\mathcal{D}(G)$ we denote the space of infinitely differentiable real-valued fuctions with compact support on $G$. If $f$ is a function on $G$ then let $f^{*}$ be the function defined by $f^{*}(x):=f\left(x^{-1}\right)$ for all $x \in G$.

Let $\mathfrak{L}(G)$ be the Lie algebra of $G$ and $\exp : \mathfrak{L}(G) \mapsto G$ the exponential mapping. An element $X \in \mathfrak{L}(G)$ can be regarded as a (left-invariant) differential operator on $G$ : for $f \in \mathcal{D}(G)$ we put

$$
X f(x)=\lim _{t \rightarrow 0} \frac{f(x \exp t X)-f(x)}{t} .
$$

Let $\left\{X_{1}, \ldots, X_{m}\right\}$ be a basis of $\mathfrak{L}(G)$. Let $x_{1}, \ldots, x_{m} \in \mathfrak{D}(G)$ be a system of skewsymmetric canonical coordinates of the first kind adapted to the basis $\left\{X_{1}, \ldots, X_{m}\right\}$ and valid in a symmetric neighbourhood $U_{0} \in \mathfrak{U}(e)$, i.e., $x_{i}^{*}=-x_{i}$ for all $i=1, \ldots, m$, $U_{0}^{-1}=U_{0}$ and

$$
y=\exp \left(\sum_{i=1}^{m} x_{i}(y) X_{i}\right) \quad \text { for each } y \in U_{0} .
$$

Let $\varphi: G \rightarrow[0,1]$ be a Hunt function for $G$, i.e., $1-\varphi \in \mathcal{D}(G)$ and

$$
\varphi(y)=\sum_{i=1}^{d} x_{i}(y)^{2} \quad \text { for all } y \in U_{0} .
$$

$\mathfrak{M}_{+}(G)$ denotes the space of positive Radon measures on $G, \mathfrak{M}_{+}^{b}(G)$ the subset of bounded positive measures on $G$ equipped with the weak topology, and $\mathfrak{M}^{1}(G)$ the subset of probability measures on $G$. The Dirac measure in $x \in G$ will be denoted by $\varepsilon_{x}$. For $\mu \in \mathfrak{M}^{1}(G)$ the adjoint measure $\widetilde{\mu}$ is defined by $\widetilde{\mu}(f):=\mu\left(f^{*}\right)$ for every continuous function $f: G \rightarrow \mathbb{C}$ with compact support. A measure $\mu \in \mathfrak{M}^{1}(G)$ is said to be normal if $\mu * \widetilde{\mu}=\widetilde{\mu} * \mu$.

For every $\mu \in \mathfrak{M}^{1}(G)$ the probability operator $T_{\mu}$ of $\mu$ is the contraction on $\mathfrak{C}^{0}(G)$ defined by

$$
T_{\mu} f(x):=\int_{G} f(x y) \mu(d y) .
$$

We will also use the important characteristic

$$
q(\mu):=\sum_{i=1}^{d}\left|\int_{G} x_{i}(y) \mu(d y)\right|+\int_{G} \varphi(y) \mu(d y) .
$$

A family $\left(\mu_{t}\right)_{t \geqslant 0}$ in $\mathfrak{M}^{1}(G)$ is called a (continuous) convolution semigroup if $\mu_{s} * \mu_{t}=$ $\mu_{s+t}$ for all $s, t \in \mathbb{R}_{+}, \mu_{0}=\varepsilon_{e}$ and $\mathcal{T}_{w^{-}} \lim _{t \downarrow 0} \mu_{t}=\mu_{0}$. Its (infinitesimal) generator is 
defined by

$$
N f:=\|\cdot\|_{\infty}-\lim _{t \downarrow 0} \frac{1}{t}\left(T_{\mu_{t}} f-f\right)
$$

for all $f$ in the maximal domain $\operatorname{Dom}(N)$ of $N$. Obviously $\left(T_{\mu_{t}}\right)_{t \geqslant 0}$ is an operator semigroup of contractions on $\mathfrak{e}^{0}(G)$ and $N$ is its generator.

A convolution semigroup $\left(\mu_{t}\right)_{t \geqslant 0}$ of non-degenerate measures is called a Gauss semigroup if $\lim _{t \downarrow 0} t^{-1} \mu_{t}(\complement U)=0$ for all $U \in \mathfrak{U}(e)$. A non-degenerate convolution semigroup $\left(\mu_{t}\right)_{t \geqslant 0}$ is a Gauss semigroup if and only if its generator has the form

$$
N=\sum_{i=1}^{m} a_{i} X_{i}+\frac{1}{2} \sum_{i, j=1}^{m} b_{i j} X_{i} X_{j}
$$

where $\left(a_{1}, \ldots, a_{m}\right) \in \mathbb{R}^{m}$ and $\left(b_{i j}\right)_{1 \leqslant i, j \leqslant m}$ is a real symmetric positive semidefinite matrix. A measure $\mu \in \mathfrak{M}^{1}(G)$ is called a Gauss measure if there exists a Gauss semigroup $\left(\mu_{t}\right)_{t \geqslant 0}$ such that $\mu_{1}=\mu$.

For $\gamma \in \mathfrak{M}_{+}^{b}(G)$ the Poisson measure $\exp \left(\gamma-\gamma(G) \varepsilon_{e}\right) \in \mathfrak{M}^{1}(G)$ with exponent $\gamma$ is defined by

$$
\exp \left(\gamma-\gamma(G) \varepsilon_{e}\right):=e^{-\gamma(G)} \sum_{k=0}^{\infty} \frac{\gamma^{k}}{k !}
$$

where $\gamma^{k}$ is the $k^{\text {th }}$ convolution power of $\gamma$ and $\gamma^{0}:=\varepsilon_{e}$.

A convolution semigroup $\left(\nu_{t}\right)_{t \geqslant 0}$ is said to be normal if for all $t \geqslant 0$ the measure $\nu_{t}$ is normal.

\section{Unitary representations and Fourier transforms}

A (continuous) unitary representation of $G$ is a homomorphism $D$ of $G$ into the group of unitary operators on a complex Hilbert space $\mathcal{H}$ such that the mapping $x \rightarrow D(x) u$ of $G$ into $\mathcal{H}$ is continuous for all $u \in \mathcal{H}$. The space $\mathcal{H}$ is called the representation space of $D$ and is denoted by $\mathcal{H}(D)$. The inner product and the norm in $\mathcal{H}(D)$ are denoted by $\langle\cdot, \cdot\rangle$ and $\|\cdot\|$, respectively. The class of all unitary representations of $G$ is denoted by $\operatorname{Rep}(G)$. By $\operatorname{Irr}(G)$ we denote the class of all irreducible representations in $\operatorname{Rep}(G)$.

Let $D \in \operatorname{Rep}(G)$. The vector $u \in \mathcal{H}(D)$ is said to be differentiable for $D$ if the coefficient function $x \rightarrow\langle D(x) u, v\rangle$ of $G$ into $\mathbb{C}$ is in $\mathcal{E}(G)$ for all $v \in \mathcal{H}(D)$. By $\mathcal{H}_{0}(D)$ we denote the space of all vectors in $\mathcal{H}(D)$ differentiable for $D$. For $X \in \mathfrak{L}(G)$ and $D \in \operatorname{Rep}(G)$ let $X(D)$ be the linear operator on $\mathcal{H}_{0}(D)$ defined by

$$
X(D) u:=\lim _{t \rightarrow 0} \frac{1}{t}(D(\exp t X)-D(e)) u
$$


see, e.g., Siebert [6].

For a probability measure $\mu$ on $G$ we define its Fourier transform $\widehat{\mu}$ by

$$
\langle\widehat{\mu}(D) u, v\rangle=\int_{G}\langle D(x) u, v\rangle \mu(d x)
$$

for all $D \in \operatorname{Rep}(G), u, v \in \mathcal{H}(D)$. Then $\widehat{\mu}(D)$ is a bounded linear operator on $\mathcal{H}(D)$. (For information on Fourier transforms see Heyer [2], Siebert [6].)

The following lemma is the key in order to apply accompanying Poisson system of a triangular system of probability measures.

3.1 Lemma. Let $\mu$ be a probability measure on a Lie group $G$. Then for all $D \in \operatorname{Rep}(G)$ and $u \in \mathcal{H}_{0}(D)$ there exists a constant $c(D, u)$ such that

$$
\|\widehat{\mu}(D) u-u\| \leqslant c(D, u) q(\mu) .
$$

Proof. By Lemma 5.1 in Siebert [6] the following Taylor formula is valid: for all $D \in \operatorname{Rep}(G), u \in \mathcal{H}_{0}(D)$ and $y \in U_{0}$

$$
D(y) u=u+\sum_{i=1}^{m} x_{i}(y) X_{i}(D) u+\frac{1}{2} \sum_{i, j=1}^{m} x_{i}(y) x_{j}(y) T(D)(y) X_{i}(D) X_{j}(D) u
$$

where $T(D)(y)$ is a bounded linear operator on $\mathcal{H}(D)$ with $\|T(D)(y)\| \leqslant 1$. Hence

$$
\begin{aligned}
\left\|\int_{G}(D(y) u-u) \mu(d y)\right\| \leqslant & 2\|u\| \mu\left(\complement U_{0}\right)+\sum_{i=1}^{m}\left\|X_{i}(D) u\right\| \cdot\left|\int_{U_{0}} x_{i}(y) \mu(d y)\right| \\
& +\frac{1}{2} \sum_{i, j=1}^{m}\left\|X_{i}(D) X_{j}(D) u\right\| \cdot \int_{U_{0}}\left|x_{i}(y) x_{j}(y)\right| \mu(d y) .
\end{aligned}
$$

Obviously

$$
\left|\int_{U_{0}} x_{i}(y) \mu(d y)\right| \leqslant\left|\int_{G} x_{i}(y) \mu(d y)\right|+\left\|x_{i}\right\|_{\infty} \cdot \mu\left(\complement U_{0}\right) .
$$

Further, we have $\left|x_{i}(y) x_{j}(y)\right| \leqslant \varphi(y)$ for all $y \in U_{0}, i, j=1, \ldots, m$, thus

$$
\int_{U_{0}}\left|x_{i}(y) x_{j}(y)\right| \mu(d y) \leqslant \int_{G} \varphi(y) d \mu(d y) .
$$

Moreover there exists a constant $c>0$ such that ${ }^{1} \complement_{U_{0}} \leqslant c \cdot \varphi$, consequently

$$
\mu\left(\complement U_{0}\right) \leqslant c \int_{G} \varphi(y) d \mu(d y),
$$

hence the proof is complete. 


\section{Triangular systems of probability measures}

A triangular system $\mathcal{I}=\left(\mu_{n \ell}\right)_{\ell=1, \ldots, k_{n} ; n \geqslant 1}$ of probability measures on $G$ is called infinitesimal if

$$
\lim _{n \rightarrow \infty} \max _{1 \leqslant \ell \leqslant k_{n}} \mu_{n \ell}(\complement U)=0 \quad \text { for all } U \in \mathfrak{U}(e) .
$$

$\mathcal{I}$ is said to be commutative if we have

$$
\mu_{n i} * \mu_{n j}=\mu_{n j} * \mu_{n i} \quad \text { for all } 1 \leqslant i, j \leqslant k_{n}, n \geqslant 1
$$

$\mathcal{I}$ is said to be normal if we have

$$
\mu_{n i} * \widetilde{\mu}_{n j}=\widetilde{\mu}_{n j} * \mu_{n i} \quad \text { for all } 1 \leqslant i, j \leqslant k_{n}, \quad n \geqslant 1
$$

$\mathcal{I}$ is said to be convergent with limit $\mu$ if we have

$$
\mu \in \mathfrak{M}^{1}(G) \quad \text { and } \quad \lim _{n \rightarrow \infty} \mu_{n 1} * \cdots * \mu_{n k_{n}}=\mu \text {. }
$$

The accompanying Poisson system $\mathcal{I}_{a}=\left(\nu_{n \ell}\right)_{\ell=1, \ldots, k_{n} ; n \geqslant 1}$ of $\mathcal{I}$ is defined by

$$
\nu_{n \ell}:=\exp \left(\mu_{n \ell}-\varepsilon_{e}\right), \quad \ell=1, \ldots, k_{n}, \quad n \geqslant 1
$$

If $\mathcal{I}$ is commutative then $\mathcal{I}_{a}$ is also commutative and the row products of $\mathcal{I}_{a}$ are the Poisson measures

$$
\exp \left(\sum_{\ell=1}^{k_{n}}\left(\mu_{n \ell}-\varepsilon_{e}\right)\right), \quad n \geqslant 1
$$

Now let $\mathcal{I}=\left(\mu_{n \ell}\right)_{\ell=1, \ldots, k_{n} ; n \geqslant 1}$ be a triangular system of probability measures on $G$. Let us consider the following condition:

(B) $\sup _{n \geqslant 1} \sum_{\ell=1}^{k_{n}}\left\|\widehat{\mu}_{n \ell}(D) u-u\right\|<\infty \quad$ for all $D \in \operatorname{Irr}(G), u \in \mathcal{H}_{0}(D)$.

Siebert [6, Proposition 8.1] has proved that if $\mathcal{I}$ is an infinitesimal and commutative triangular system satisfying condition $(\mathrm{B})$ then the system $\mathcal{I}$ is convergent if and only if the accompanying Poisson system $\mathcal{I}_{a}$ is convergent, and in the affirmative case their limits coincide.

\section{Lindeberg-Feller theorems on Lie groups}

First we prove a convergence theorem for triangular systems of symmetric probability measures. 
5.1 Theorem. Let $\mathcal{I}=\left(\mu_{n \ell}\right)_{\ell=1, \ldots, k_{n} ; n \geqslant 1}$ be a commutative system of symmetric probability measures on a Lie group $G$. Let $\left(b_{i j}\right)_{i, j=1, \ldots, m}$ be a real symmetric positive semidefinite matrix.

Then the following statements are equivalent:

(i) (a) $\lim _{n \rightarrow \infty} \sum_{\ell=1}^{k_{n}} \mu_{n \ell}(\complement U)=0$ for all $U \in \mathfrak{U}(e)$,

(b) $\lim _{n \rightarrow \infty} \sum_{\ell=1}^{k_{n}} \int_{G} x_{i}(y) x_{j}(y) \mu_{n \ell}(d y)=b_{i j}$ for all $i, j=1, \ldots, m$.

(ii) (a) $\mathcal{I}$ is infinitesimal,

(b) $\sup _{n \geqslant 1} \sum_{\ell=1}^{k_{n}} \int_{G} \varphi(y) d \mu_{n \ell}<\infty$,

(c) $\lim _{n \rightarrow \infty} \mu_{n 1} * \cdots * \mu_{n k_{n}}=\nu$ where $\nu=\nu_{1}, \quad\left(\nu_{t}\right)_{t \geqslant 0}$ is the Gauss semigroup with generator $\frac{1}{2} \sum_{i, j=1}^{m} b_{i j} X_{i} X_{j}$.

Proof. (i) $\Longrightarrow\left(\right.$ ii). Obviously (ii)(a) follows from (i)(a). The symmetry of $\mu_{n \ell}$ implies $\int_{G} x_{i}(y) \mu_{n \ell}(d y)=0$ for all $i=1, \ldots, m$, thus we have clearly

$$
q\left(\mu_{n \ell}\right)=\int_{G} \varphi(y) \mu_{n \ell}(d y) \leqslant \sum_{i=1}^{m} \int_{G} x_{i}(y)^{2} \mu_{n \ell}(d y)+\mu_{n \ell}\left(\complement U_{0}\right)
$$

Hence we obtain (ii)(b). Moreover, conditions (i)(a) and (i)(b) together with Lemma 3.1 imply that the system satisfies Condition (B), thus (ii)(c) can be derived by the help of Proposition 8.1 in Siebert [6] as in the proof of Theorem 8.1 in Pap [4].

(ii) $\Longrightarrow($ i). Condition (ii)(b) together with Lemma 3.1 imply that the system satisfies Condition (B). Again one can use the same arguments as in the proof of Theorem 8.1 in Pap [4].

Using symmetrization we obtain the following generalization.

5.2 Theorem. Let $\mathcal{I}=\left(\mu_{n \ell}\right)_{\ell=1, \ldots, k_{n} ; n \geqslant 1}$ be a commutative system of probability measures on a Lie group $G$. Let $\left(a_{1}, \ldots, a_{m}\right) \in \mathbb{R}^{m}$ and $\left(b_{i j}\right)_{i, j=1, \ldots, m}$ be a real symmetric positive semidefinite matrix.

Let us consider the following assertions:

(i) (a) $\lim _{n \rightarrow \infty} \sum_{\ell=1}^{k_{n}} \mu_{n \ell}(\complement U)=0$ for all $U \in \mathfrak{U}(e)$, 

(b) $\lim _{n \rightarrow \infty} \sum_{\ell=1}^{k_{n}} \int x_{i}(y) x_{j}(y) \mu_{n \ell}(d y)=b_{i j}$ for all $i, j=1, \ldots, m$,
(c) $\lim _{n \rightarrow \infty} \sum_{\ell=1}^{k_{n}} \int x_{i}(y) \mu_{n \ell}(d y)=a_{i}$ for all $i=1, \ldots, m$,
(d) $\sup _{n \geqslant 1} \sum_{\ell=1}^{k_{n}}\left|\int x_{i}(y) \mu_{n \ell}(d y)\right|<\infty$ for all $i=1, \ldots, m$.

(ii) (a) $\mathcal{I}$ is infinitesimal,

(b) $\sup _{n \geqslant 1} \sum_{\ell=1}^{k_{n}} q\left(\mu_{n \ell}\right)<\infty$,

(c) $\lim _{n \rightarrow \infty} \mu_{n 1} * \cdots * \mu_{n k_{n}}=\nu$ where $\nu=\nu_{1}, \quad\left(\nu_{t}\right)_{t \geqslant 0}$ is the Gauss semigroup with generator $\sum_{i=1}^{m} a_{i} X_{i}+\frac{1}{2} \sum_{i, j=1}^{m} b_{i j} X_{i} X_{j}$.

Then (i) implies (ii).

If we suppose that in addition the triangular system $\mathcal{I}$ is normal and the semigroup $\left(\nu_{t}\right)_{t \geqslant 0}$ is normal and uniquely determined by $\nu_{1}$ then (i) and (ii) are equivalent.

Proof. (i) $\Longrightarrow$ (ii). Conditions (a), (b) and (d) of (i) together with Lemma 3.1 imply that the system satisfies Condition (B). Then one can repeat the arguments of the proof of Theorem 8.2 in Pap [4].

(ii) $\Longrightarrow$ (i). Again condition (ii)(b) together with Lemma 3.1 imply that the system satisfies Condition (B), hence we can use the arguments of the proof of Theorem 8.2 in Pap [4].

5.3 Remark. The statement (i) $\Longrightarrow$ (ii) is the central limit theorem of Wehn [7].

\section{Special case: stratified Lie groups}

A Lie algebra $\mathfrak{L}(G)$ has a stratified decomposition of step $s$ if there exists a vector space decomposition $\mathfrak{L}(G)=\oplus_{k=1}^{s} V_{k}$ such that $\left[V_{k}, V_{\ell}\right] \subset V_{k+\ell}$ for $k+\ell \leqslant s, \quad\left[V_{k}, V_{\ell}\right]=\{0\}$ for $k+\ell>s$, and $V_{1}$ generates $\mathfrak{L}(G)$ as an algebra. A basis $\left\{X_{1}, \ldots, X_{m}\right\}$ of $\mathfrak{L}(G)$ is adapted to the above decomposition if for each $k=1, \ldots, s$ the basis elements in $V_{k}$ form a basis for $V_{k}$. For $i=1, \ldots, m$ let $d_{i}:=k$ if $X_{i} \in V_{k}$.

A stratified Lie group of step $s$ is a simply connected Lie group whose Lie algebra has a stratified decomposition of step $s$. Clearly a stratified Lie group of step $s$ is nilpotent of step $s$. 
If $\nu$ is a Gauss measure on a nilpotent Lie group then there exists a uniquely determined Gauss semigroup $\left(\nu_{t}\right)_{t \geqslant 0}$ such that $\nu=\nu_{1}$ (see Pap [5]). The generator of $\left(\nu_{t}\right)_{t \geqslant 0}$ will be called the generator of $\nu$.

Let $G$ be a stratified Lie group of step $s$. Let $\left\{X_{1}, \ldots, X_{m}\right\}$ be an adapted basis of its Lie algebra $\mathfrak{L}(G)$. It is known that the exponential mapping exp : $\mathfrak{L}(G) \mapsto G$ is now an analytic diffeomorphism. Let $\left\{\zeta_{1}, \ldots, \zeta_{m}\right\}$ be the global canonical coordinates of the first kind of $G$ defined by

$$
y=\exp \left(\sum_{i=1}^{m} \zeta_{i}(y) X_{i}\right) \quad \text { for all } y \in G
$$

We equip $\mathfrak{L}(G)$ and $G$ with the natural dilations by extending

$$
\stackrel{\circ}{\delta}_{t}\left(X_{i}\right)=t^{d_{i}} X_{i}, \quad t>0, \quad i=1, \ldots, m
$$

by linearity to $\mathfrak{L}(G)$ and transferring it to $G$ by

$$
\delta_{t}=\exp \circ \stackrel{\circ}{\delta}_{t} \circ \exp ^{-1}, \quad t>0
$$

A homogeneous norm on $G$ is a continuous function $y \mapsto|y|$ from $G$ into $\mathbb{R}_{+}$such that $\left|\delta_{t} y\right|=t|y|$ for all $t>0$ and $y \in G$, and $|y|=0$ if and only if $y=e$. Homogeneous norms always exist, e.g.,

$$
y \mapsto \sum_{i=1}^{m}\left|\zeta_{i}(y)\right|^{1 / d_{i}}
$$

It is known that the homogeneous norms are equivalent as norms, and for each homogeneous norm $|\cdot|$ we have

$$
\left|\zeta_{i}(y)\right| \leqslant c_{1}|y|^{d_{i}} \quad \text { for all } y \in G, \quad i=1, \ldots, m
$$

with a suitable constant $c_{1}>0$ depending only on the homogeneous norm $|\cdot|$ (cf. Folland and Stein [1], Pap [3]) .

Now we can choose local canonical coordinates $\left\{x_{1}, \ldots, x_{m}\right\}$ of the first kind in $\mathcal{D}(G)$ valid in the neighbourhood $U_{0}:=\{y \in G:|y|<1 / 2\}$ and satisfying

$$
\left|x_{i}(y)\right| \leqslant\left|\zeta_{i}(y)\right| \quad \text { for all } y \in G, \quad i=1, \ldots, m \text {. }
$$

(Obviously $x_{i}(y)=\zeta_{i}(y)$ for all $y \in U_{0}, \quad i=1, \ldots, m$.) Further we choose a Hunt function $\varphi: G \rightarrow[0,1]$. Clearly we have for all $y \in U_{0}$

$$
\varphi(y)=\sum_{i=1}^{m} x_{i}(y)^{2}=\sum_{i=1}^{m} \zeta_{i}(y)^{2} \leqslant c_{1}^{2} \sum_{i=1}^{m}|y|^{2 d_{i}} \leqslant c_{1}^{2} \sum_{i=1}^{m}|y|^{2}=m c_{1}^{2}|y|^{2} .
$$


For $y \in \complement U_{0}$ we can use simply $\varphi(y) \leqslant 1$, hence

$$
\varphi(y) \leqslant c_{2} \min \left\{|y|^{2}, 1\right\} \quad \text { for all } y \in G
$$

with a suitable constant $c_{2}>0$ depending only on the homogeneous norm $|\cdot|$. Consequently Theorem 5.1 implies the following generalization of Theorem 8.1 in Pap [4].

6.1 Theorem. Let $\mathcal{I}=\left(\mu_{n \ell}\right)_{\ell=1, \ldots, k_{n} ; n \geqslant 1}$ be a commutative system of symmetric probability measures on a stratified Lie group $G$ with a homogeneous norm $|\cdot|$. Let $\left(b_{i j}\right)_{i, j=1, \ldots, m}$ be a real symmetric positive semidefinite matrix.

Let us consider the following assertions:

(i) (a) $\lim _{n \rightarrow \infty} \sum_{\ell=1}^{k_{n}} \mu_{n \ell}(\{y \in G:|y|>\varepsilon\})=0$ for all $\varepsilon>0$,

(b) $\lim _{n \rightarrow \infty} \sum_{\ell=1}^{k_{n}} \int_{|y|<1} \zeta_{i}(y) \zeta_{j}(y) \mu_{n \ell}(d y)=b_{i j}$ for all $i, j=1, \ldots, m$.

(ii) (a) $\mathcal{I}$ is infinitesimal,

(b) $\lim _{n \rightarrow \infty} \mu_{n 1} * \cdots * \mu_{n k_{n}}=\nu$ where $\nu$ is the Gauss measure with generator

$$
\frac{1}{2} \sum_{i, j=1}^{m} b_{i j} X_{i} X_{j} \text {. }
$$

Then (i) implies (ii).

If we suppose that in addition

$$
\sup _{n \geqslant 1} \sum_{\ell=1}^{k_{n}} \int_{G} \min \left\{|y|^{2}, 1\right\} \mu_{n \ell}(d y)<\infty
$$

then (i) and (ii) are equivalent.

We have for all $i=1, \ldots, m$

$$
\left|\int_{G} x_{i}(y) \mu(d y)\right| \leqslant\left\|x_{i}\right\|_{\infty} \cdot \mu\left(\complement U_{0}\right)+\int_{U_{0}}\left|\zeta_{i}(y)\right| \mu(d y) \leqslant\left\|x_{i}\right\|_{\infty} \cdot \mu\left(\complement U_{0}\right)+c_{1} \int_{U_{0}}|y| \mu(d y),
$$

hence we conclude

$$
q(\mu) \leqslant c_{3} \int_{G} \min \{|y|, 1\} \mu(d y)
$$

with a suitable constant $c_{3}>0$ depending only on the homogeneous norm $|\cdot|$ and the local coordinates $x_{1}, \ldots, x_{m}$. Hence using Theorem 5.2 we obtain the following generalizations of Theorem 8.2 in Pap [4].

6.2 Theorem. Let $\mathcal{I}=\left(\mu_{n \ell}\right)_{\ell=1, \ldots, k_{n} ; n \geqslant 1}$ be a commutative system of probability measures on a stratified Lie group $G$ with a homogeneous norm $|\cdot|$. Let $\left(a_{1}, \ldots, a_{m}\right) \in$ $\mathbb{R}^{m}$ and $\left(b_{i j}\right)_{i, j=1, \ldots, m}$ be a real symmetric positive semidefinite matrix. 
Let us consider the following assertions:

(i) (a) $\lim _{n \rightarrow \infty} \sum_{\ell=1}^{k_{n}} \mu_{n \ell}(\{y \in G:|y|>\varepsilon\})=0$ for all $\varepsilon>0$,

(b) $\lim _{n \rightarrow \infty} \sum_{\ell=1}^{k_{n}} \int_{|y|<1} \zeta_{i}(y) \zeta_{j}(y) \mu_{n \ell}(d y)=b_{i j}$ for all $i, j=1, \ldots, m$,

(c) $\lim _{n \rightarrow \infty} \sum_{\ell=1}^{k_{n}} \int_{|y|<1} \zeta_{i}(y) \mu_{n \ell}(d y)=a_{i}$ for all $i=1, \ldots, m$

(d) $\sup _{n \geqslant 1} \sum_{\ell=1}^{k_{n}}\left|\int_{|y|<1} \zeta_{i}(y) \mu_{n \ell}(d y)\right|<\infty$ for all $i=1, \ldots, m$, .

(ii) (a) $\mathcal{I}$ is infinitesimal,

(b) $\lim _{n \rightarrow \infty} \mu_{n 1} * \cdots * \mu_{n k_{n}}=\nu$ where $\nu$ is the Gauss measure with generator

$$
\sum_{i=1}^{m} a_{i} X_{i}+\frac{1}{2} \sum_{i, j=1}^{m} b_{i j} X_{i} X_{j}
$$

Then (i) implies (ii).

If we suppose that in addition the triangular system $\mathcal{I}$ and the Gauss semigroup $\left(\nu_{t}\right)_{t \geqslant 0}$ with $\nu_{1}=\nu$ are normal, and

$$
\sup _{n \geqslant 1} \sum_{\ell=1}^{k_{n}} \int_{G} \min \{|y|, 1\} \mu_{n \ell}(d y)<\infty
$$

then (i) and (ii) are equivalent.

Note that Theorem 6.1 and 6.2 are not only generalizations but also improvements of Theorem 8.1 and 8.2 in Pap [4], respectively, which were proved under the condition

$$
\sup _{n \geqslant 1} \sum_{\ell=1}^{k_{n}} \int_{G}|y|^{2} \mu(d y)<\infty .
$$

If we suppose convergence of the covariance matrices corresponding only to the coordinates $\zeta_{i}$ with $d_{i}=1$ and the Lindeberg condition for the coordinates $\zeta_{i}$ with $d_{i} \in\{2, \ldots, s\}$ then we obtain the usual form of the Lindeberg-Feller theorem, which is a generalization of Theorem 8.3 in [4]. For the sake of simplicity we formulate the result inly in case of centered measures. A probability measure $\mu$ on a stratified Lie group $G$ is called centered if

$$
\int_{G} \zeta_{i}(y) \mu(d y)=0 \quad \text { whenever } \quad d_{i}=1 .
$$

6.3 Theorem. Let $\mathcal{I}=\left(\mu_{n \ell}\right)_{\ell=1, \ldots, k_{n} ; n \geqslant 1}$ be a commutative and normal system of centered probability measures on a stratified Lie group $G$ with a homogeneous norm $|\cdot|$. 
Let $\left(b_{i j}\right)_{i, j=1, \ldots, m}$ be a real symmetric positive semidefinite matrix. Suppose that the Gauss semigroup $\left(\nu_{t}\right)_{t \geqslant 0}$ with generator

$$
\frac{1}{2} \sum_{d_{i}=d_{j}=1}^{m} b_{i j} X_{i} X_{j}
$$

is normal. Suppose that $\mathcal{I}$ satisfies the conditions

$$
\begin{aligned}
\sup _{n \geqslant 1} \sum_{\ell=1}^{k_{n}} \int_{|y|<1}\left|\zeta_{i}(y)\right|^{2 / d_{i}} \mu_{n \ell}(d y) & <\infty \quad \text { whenever } d_{i} \in\{2, \ldots, s\}, \\
\lim _{n \rightarrow \infty} \sum_{\ell=1}^{k_{n}} \int_{|y| \geqslant 1}\left|\zeta_{i}(y)\right|^{2 / d_{i}} \mu_{n \ell}(d y) & =0 \quad \text { whenever } d_{i} \in\{2, \ldots, s\}, \\
\lim _{n \rightarrow \infty} \sum_{\ell=1}^{k_{n}} \int \zeta_{i}(y) \mu_{n \ell}(d y) & =0 \quad \text { whenever } d_{i} \in\{2, \ldots, s\}, \\
\lim _{n \rightarrow \infty} \sum_{\ell=1}^{k_{n}} \int \zeta_{i}(y) \zeta_{j}(y) \mu_{n \ell}(d y) & =0 \quad \text { whenever } d_{i}=d_{j}=1 .
\end{aligned}
$$

Then the following statements are equivalent:

(i) (a) $\lim _{n \rightarrow \infty} \max _{1 \leqslant \ell \leqslant k_{n}} \int|y|^{2} \mu_{n \ell}(d y)=0$,

(b) $\lim _{n \rightarrow \infty} \mu_{n 1} * \cdots * \mu_{n k_{n}}=\nu_{1}$.

(ii) (a) $\lim _{n \rightarrow \infty} \sum_{\ell=1}^{k_{n}} \mu_{n \ell}(\{y \in G:|y|>\varepsilon\})=0$ for all $\varepsilon>0$,

(b) $\lim _{n \rightarrow \infty} \sum_{\ell=1}^{k_{n}} \int_{|y|<1} \zeta_{i}(y) \zeta_{j}(y) \mu_{n \ell}(d y)=b_{i j} \quad$ whenever $d_{i}=d_{j}=1$.

(iii) $\lim _{n \rightarrow \infty} \sum_{\ell=1}^{k_{n}} \int_{|y| \geqslant \varepsilon}|y|^{2} \mu_{n \ell}(d y)=0$ for all $\varepsilon>0$.

\section{References}

[1] Folland, G. B. and Stein, E. M. (1982). Hardy spaces on homogeneous groups. Princeton University Press, New Jersey.

[2] Heyer, H. (1977). Probability Measures on Locally Compact Groups. Springer, Berlin Heidelberg New York. 
[3] PAP, G. (1991). Rate of convergence in CLT on stratified groups. J. Multivariate Anal. 38, 333-365.

[4] PAP, G. (1993). Central limit theorems on nilpotent Lie groups. Probability and Mathematical Statistics 14, 287-312.

[5] PAP, G. (1994). Uniqueness of embedding into a Gaussian semigroup on a nilpotent Lie group. Archiv der Math. 49, 282-288.

[6] Siebert, E. (1981). Fourier analysis and limit theorems for convolution semigroups on a locally compact group. Adv. Math. 39, 111-154.

[7] Wehn, D. (1962). Probabilities on Lie groups. Proc. Natl. Acad. Sci. USA 48, 791795. 